\title{
(6) OPEN ACCESS \\ Abnormal breathing of sudden cardiac arrest victims described by laypersons and its association with emergency medical service dispatcher-assisted cardiopulmonary resuscitation instruction
}

\author{
Hidetada Fukushima, ${ }^{1}$ Masami Imanishi, ${ }^{2}$ Taku Iwami, ${ }^{3}$ Tadahiko Seki, ${ }^{1}$ \\ Yasuyuki Kawai, ${ }^{1}$ Kazunobu Norimoto, ${ }^{1}$ Yasuyuki Urisono, ${ }^{1}$ Michiaki Hata, ${ }^{1}$ \\ Kenji Nishio, ${ }^{4}$ Keigo Saeki, ${ }^{5}$ Norio Kurumatani, ${ }^{5}$ Kazuo Okuchi ${ }^{1}$
}

${ }^{1}$ Department of Emergency and Critical Care Medicine, Nara Medical University, Kashihara, Nara, Japan

${ }^{2}$ Department of Neurosurgery, Nara Saiseikai Gose Hospital, Gose, Nara, Japan

${ }^{3}$ Department of Health Service, Kyoto University Health Service, Kyoto, Kyoto, Japan ${ }^{4}$ Department of General Medicine, Nara Medical University, Kashihara, Nara, Japan

${ }^{5}$ Department of Community Health and Epidemiology, Nara Medical University, Kashihara, Nara, Japan

\section{Correspondence to}

Dr Hidetada Fukushima, Department of Emergency and Critical Care Medicine, Nara Medical University, Shijo-cho 840, Kashihara City, Nara 634, Japan; hidetakarina@gmail.com

Received 8 August 2013 Revised 18 October 2013 Accepted 5 December 2013 Published Online First

8 January 2014

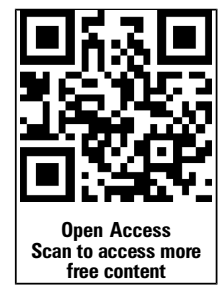

CrossMark

To cite: Fukushima $\mathrm{H}_{\text {, }}$ Imanishi $\mathrm{M}$, Iwami $\mathrm{T}$, et al. Emerg Med J 2015;32 314-317.

\section{ABSTRACT}

Background Current guidelines for cardiopulmonary resuscitation (CPR) emphasise that emergency medical service (EMS) dispatchers should identify sudden cardiac arrest (CA) with abnormal breathing and assist lay rescuers performing CPR. However, lay rescuers description of abnormal breathing may be inconsistent, and it is unclear how EMS dispatchers provide instruction for CPR based on the breathing status of the CA victims described by laypersons.

Methods and results To investigate the incidence of abnormal breathing and the association between the EMS dispatcher-assisted CPR instruction and layperson CPR, we retrospectively analysed 283 witnessed CA cases whose information regarding breathing status of CA victims was available from population-based prospective cohort data. In 169 cases (59.7\%), laypersons described that the CA victims were breathing in various ways, and that the victims were 'not breathing' in 114 cases (40.3\%). Victims described as breathing in various ways were provided EMS dispatchinstruction for CPR less frequently than victims described as 'not breathing' (27.8\% (47/169) vs $84.2 \%$ (96/114); $\mathrm{p}<0.001)$. Multivariate logistic regression showed that EMS dispatch-instruction for CPR was associated significantly with layperson CPR (adjusted OR, 11.0; $95 \% \mathrm{Cl}, 5.72$ to 21.2 ).

Conclusions This population-based study indicates that $60 \%$ of CA victims showed agonal respiration, which was described as breathing in various ways at the time of EMS call. Although EMS dispatch-instruction was associated significantly with an increase in layperson CPR, abnormal breathing was associated with a much lower rate of CPR instruction and, in turn, was related to a much lower rate of bystander CPR.

\section{INTRODUCTION}

Sudden cardiac arrest (CA) is a leading cause of death in the industrialised world. Approximately 300000 CAs in the USA and 100000 in Japan occur annually in out-of-hospital settings, and the vast majority of these victims do not survive. ${ }^{1} 2$ Rapid initiation of cardiopulmonary resuscitation (CPR) for CA victims can increase the chance of survival without neurological deficits. ${ }^{2}{ }^{3}$ However, the incidence of layperson CPR is low. ${ }^{12}$

Emergency medical service (EMS) dispatchers play a key role in the performance of CPR prior to the arrival of EMS personnel on the scene. ${ }^{4} 5$ EMS dispatchers can help laypersons identify CA and assist in the performance of CPR. However, the identification of CA victims with agonal respiration via telephone may be extremely difficult, ${ }^{6-8}$ and EMS dispatcher-assisted CPR instruction is underused. $^{7} 9$ To increase EMS dispatcher-assisted CPR instruction, we examined the actions of regional EMS dispatchers based on the description of sudden $\mathrm{CA}$ victims by laypersons. Although several studies have investigated the accuracy of CA recognition by EMS dispatchers, ${ }^{9} \quad 10$ we identified only two population-based studies of laypersons' descriptions of agonal respiration and its association with EMS dispatcher-assisted CPR instruction. ${ }^{6} 11$

In this study, we investigate laypersons' descriptions of the respiratory condition in CA victims by use of a population-based registry of out-of-hospital cardiac arrest (OHCA). Furthermore, we assessed how EMS dispatch-instruction for CPR and breathing status of the CA victims described by laypersons were associated with CPR by lay rescuers.

\section{MATERIALS AND METHODS}

We conducted a retrospective analysis of prospective cohort data of all OHCA cases aged 18 years or older in whom resuscitation was attempted and who were then transported to medical institutions from 1 January 2007 through 31 December 2009. We included OHCA cases that were witnessed by a layperson. OHCA cases that collapsed after emergency call were excluded from this analysis. This study was approved by the ethical committee of Nara Medical University.

The population of Nara Prefecture is approximately 1.4 million inhabitants in an area of around $3700 \mathrm{~km}^{2}$. Nara Prefecture has 13 fire stations with dispatch centres. The free emergency telephone number, 119, is used to call for an ambulance. All EMS dispatchers are trained firefighters. Each fire station had their own dispatch protocols for CA based on 2005 CPR guidelines. To identify CA victims, EMS dispatchers asked 119 callers whether the unresponsive victim was breathing. When the caller answered that the victim was breathing, EMS dispatchers asked how the victim was breathing and considered the possibility of agonal respiration. Once CA was suspected, the dispatchers instructed the caller to perform chest compressions or 
conventional CPR. Dispatchers did not ask laypersons to check the pulse of the victims.

Data were prospectively collected by use of a form based on the Utstein-style reporting guidelines for OHCA, ${ }^{12}{ }^{13}$ including age, sex, origin of CA, location of arrest, disabilities in daily living, EMS dispatcher-assisted CPR instruction, bystander-initiated CPR, first documented rhythm, time course of resuscitation, advanced airway management, intravenous fluids and epinephrine, as well as prehospital return of spontaneous circulation, 1-month survival and neurological status at 1 month after the event. Both chest compression-only CPR and conventional CPR with rescue breathing were considered as layperson CPR. Rescue breathing without chest compression was classified as no CPR. The outcome was assessed by the health style according to the Glasgow-Pittsburgh overall performance category (OPC) ${ }^{12}{ }^{13}$ at 1 month after the event. Good neurological outcome was defined as OPC 1 or 2.

Along with those Utstein data, we collected information regarding how laypersons described the breathing styles of CA victims. We collected the data from the anonymous written reports of emergency calls composed by each EMS dispatcher who actually took the emergency calls. EMS dispatchers asked for the callers for details of the victims' responsiveness and breathing status routinely and recorded it on the report form.

Data were presented as medians and IQRs for continuous variables and numbers and percentages for categorical variables. Groups were compared using Mann-Whitney $U$ test and $\chi^{2}$ test. Multiple logistic regression analysis assessed the factors associated with layperson-performance of CPR; adjusted ORs (AORs) and their 95\% CIs were calculated. Potential confounding factors that were biologically essential or significantly associated with layperson-performance of CPR at $\mathrm{p}<0.1$ in the univariate analyses were considered in the multivariate analyses. All statistical analyses were two-sided and performed using computer software (SPSS V.19, Illinois, USA). The results were considered to be statistically significant at a $\mathrm{p}$ value less than 0.05 .

\section{RESULTS}

During the 3-year period, EMS attempted resuscitation in 3173 OHCA cases. Of those, 735 cases met our inclusion criteria. Among these cases, layperson CPR was started before EMS calls in 76 cases. In the remaining 659 cases, information of breathing styles was not available in 376 cases, because the callers were upset, excited or separated from the victims. Therefore, we investigated 283 cases with no CPR by laypersons at the time of EMS call and with information on breathing styles (figure 1). Baseline characteristics of study subjects are described in table 1 . The characteristics of the included 283 cases and excluded 376 cases were similar, although victims with information on breathing styles collapsed more often at home than the excluded cases $(89.8 \%$ $(254 / 283)$ vs $79.0 \%(297 / 376), \mathrm{p}<0.001)$.

Laypersons described that victims were breathing in 169 cases $(59.7 \%)$ at the time of the EMS calls. Among these, victims were described as 'having difficulties in breathing' in 37 cases (13.1\%), 'weak breathing' in 30 cases (10.6\%), 'snoring' in 22 cases $(7.8 \%)$ and others in 18 cases (6.3\%). Laypersons could not determine whether the victims were breathing normally in 62 cases $(21.9 \%)$.

Baseline characteristics according to EMS dispatch-instruction for CPR are shown in table 2. Layperson CPR was performed more often in the group with EMS instruction than in the group without EMS instruction (56.6\% (81/143) vs 12.1\% (17/ 140); $\mathrm{p}<0.001)$. However, victims described as breathing in various ways were provided EMS dispatch-instruction for CPR less frequently than victims described as 'not breathing' $(27.8 \%$ $(47 / 169)$ vs $84.2 \%(96 / 114) ; \mathrm{p}<0.001)$.

\section{EMS resuscitation attempted $(\mathrm{N}=3173)$}

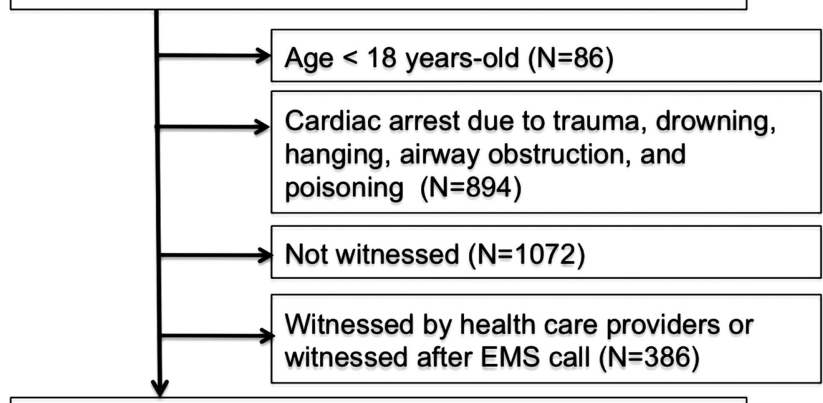

Layperson witnessed cardiac arrest $(\mathrm{N}=735)$

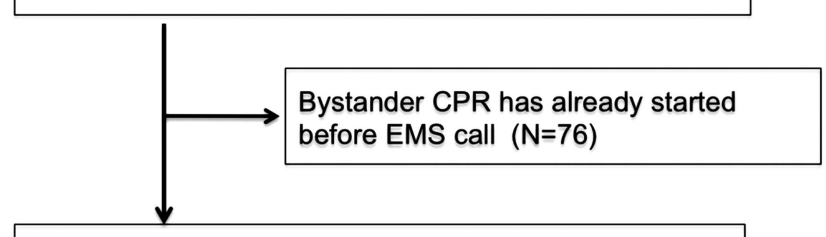

Cardiac arrest without Bystander at EMS call $(\mathrm{N}=659)$

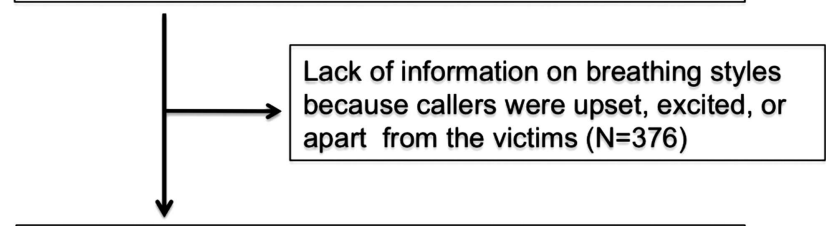

Information on breathing style at first call available $(\mathrm{N}=283)$

Figure 1 Study population. CPR, cardiopulmonary resuscitation; EMS, emergency medical service.

Table 3 shows the AORs and their 95\% CIs for layperson CPR. EMS dispatcher-assisted CPR instruction (AOR; 11.0, 95\% CI 5.72 to 21.2) and disabilities in daily living (AOR; $2.57,95 \%$ CI 1.10 to 5.99 ) were significantly associated with the increment of layperson CPR.

\section{DISCUSSION}

This study revealed that lay rescuers described as much as $60 \%$ of CA victims were exhibiting various types of breathing at their EMS call, and that EMS dispatcher-assisted CPR instruction can increase layperson CPR. The combination of a population-based Utstein style registry and detailed reports regarding breathing style of sudden CA victims allows the evaluation of the effectiveness of EMS dispatch instruction to increase CPR by laypersons considering the impact of rescuers' recognition of CA.

Our results suggest that the majority of CA victims showed abnormal breathing at the time of the EMS call. Since we excluded unwitnessed CA cases and victims who collapsed after EMS calls from all EMS resuscitation attempted CAs, these descriptions were considered to be agonal respiration. Bobrow et $a l^{14}$ reported that the presence of agonal respiration was 33\% in victims arrested after EMS arrival, and that its incidence decreased as collapse-EMS-arrival time increased. Our data indicate the incidence of agonal respiration is much greater than expected in the early phase of out-of-hospital settings where lay rescuers face sudden CA and strongly supports the current 2010 guideline for CPR emphasising the importance of dispatchers to help lay rescuers recognise agonal respiration and start CPR. 
Table 1 Baseline characteristics of the study groups

\begin{tabular}{|c|c|c|c|c|c|c|}
\hline \multirow{3}{*}{$\begin{array}{l}\text { Characteristics } \\
\text { Men, n (\%) }\end{array}$} & \multirow{2}{*}{\multicolumn{2}{|c|}{$\frac{\text { Total }}{(\mathrm{N}=659)}$}} & \multirow{2}{*}{\multicolumn{2}{|c|}{$\begin{array}{l}\text { Breathing information } \\
\text { available } \\
(\mathrm{N}=283)\end{array}$}} & \multirow{2}{*}{\multicolumn{2}{|c|}{$\begin{array}{l}\text { Breathing information } \\
\text { not available } \\
(\mathrm{N}=376)\end{array}$}} \\
\hline & & & & & & \\
\hline & 408 & $(61.9)$ & 174 & $(61.5)$ & 234 & $(62.2)$ \\
\hline Age, years, median (IQR) & 76 & $(66-84)$ & 76 & $(68-84)$ & 76 & $(65-83)$ \\
\hline Victims with disabilities in daily life, $n(\%)$ & 76 & $(20.2)$ & 36 & $(12.7)$ & 40 & $(10.6)$ \\
\hline Cardiac arrest at home, $\mathrm{n}(\%)$ & 551 & $(83.6)$ & $254^{*}$ & $(89.8)$ & 297 & $(79.0)$ \\
\hline Victims described as breathing in various ways, $n(\%)$ & - & - & 169 & $(59.7)$ & - & - \\
\hline Initial rhythm of VF/VT, n (\%) & 113 & $(17.1)$ & 43 & $(15.2)$ & 70 & $(18.6)$ \\
\hline Call-arrival time, min, median (IQR) & 7 & $(5-10)$ & 7 & $(6-9)$ & 7 & $(5-10)$ \\
\hline Advanced airway management by EMS, n (\%) & 449 & $(68.1)$ & 204 & $(72.1)$ & 245 & $(65.2)$ \\
\hline Epinephrine administration by EMS, n (\%) & 83 & $(12.6)$ & 42 & $(14.8)$ & 41 & $(10.9)$ \\
\hline Time from call to hospital arrival, min, median (IQR) & 35 & $(28-44)$ & 35 & $(29-44)$ & 35 & $(27-44)$ \\
\hline
\end{tabular}

This study also showed that laypersons describe agonal respiration in a wide variety of ways such as weak breathing or difficulties in breathing. Since the descriptions of agonal respirations are diverse, it is difficult for dispatchers to distinguish true CA at emergency calls. In this study, while $84.2 \%$ (96 out of 114 cases) of those who were described as 'not breathing' were identified as CA and provided CPR instruction, only 27.8\% (47 of 169 cases) of those with possible agonal respiration described as various types of breathing were identified as CA and provided CPR instruction by the EMS dispatchers. Along with previous reports, ${ }^{6}{ }^{7}$ many $\mathrm{CA}$ victims with agonal respirations might lose the chance to receive CPR because of the misrecognition of CA. In a literature review, identifying $\mathrm{CA}$ by a combination of 'unconsciousness' and 'absence of breathing' or the 'presence of abnormal breathing' was shown to have a high sensitivity and specificity. ${ }^{15-18}$ However, the sensitivity and specificity of this protocol have not been explored fully. ${ }^{19}$ A large observational study analysing about 10000 emergency calls reported that 'abnormal breathing' could not differentiate CA. ${ }^{8}$ Additional information such as victims' facial colour, or coldness of the body, ${ }^{8}$ or asking the callers to state 'now' each time the victim takes a breath ${ }^{20}$ would help EMS dispatchers to identify CA. However, the time to collect additional information is limited for EMS dispatchers, and it should be noted that delayed recognition of CA and CPR relate to poor prognosis. ${ }^{21}$ In addition, since victims not in $\mathrm{CA}$ such as stroke can present abnormal breathing, ${ }^{22}$ there are concerns about the risk of layperson CPR on non-CA victims. ${ }^{23}$ However, recent studies showed that the frequency of serious injury on non-CA victims by CPR was very low. $^{24} 25$ Considering the high frequency of agonal respiration, difficulties in differentiating CA by lay rescuers' descriptions of abnormal breathing and low risk of severe injury by CPR on non-CA victims, an active protocol for dispatchers to encourage lay rescuers to perform CPR presupposing an overdiagnosis of CA should be considered.

Multivariate analysis demonstrated that EMS dispatcher instruction for CPR was significantly associated with an increase in layperson CPR. However, this study also demonstrated that abnormal breathing was associated with a much lower rate of CPR instruction and, in turn, was related to a much lower rate of bystander CPR. These findings, along with the data regarding breathing status in sudden CA victims described by laypersons, emphasise the role of EMS dispatchers to identify CA victims with possible agonal respiration via telephone for improving the chain of survival.

\section{LIMITATIONS}

There are inherent limitations in this study.

First, over half of the eligible patients were excluded from the study because there was no information about breathing status. The callers who could report the victims' breathing status might be acting more calm than callers in the excluded group and there might be a bias in laypersons' descriptions of breathing status. This is a substantial limitation. However, because EMS dispatchers were required to ask and record the victims' responsiveness and breathing status from the callers routinely, this low proportion of cases with complete information on breathing status suggests that it is usual for the callers to be upset, excited

Table 2 Layperson CPR and outcomes according to EMS dispatcher-assisted CPR instruction

\begin{tabular}{|c|c|c|c|c|c|c|}
\hline \multirow[b]{2}{*}{ Characteristics } & \multicolumn{2}{|c|}{ Total } & \multicolumn{2}{|c|}{$\begin{array}{l}\text { EMS dispatch-instruction } \\
\text { for CPR }(+)\end{array}$} & \multicolumn{2}{|c|}{$\begin{array}{l}\text { EMS dispatch-instruction } \\
\text { for CPR (-) }\end{array}$} \\
\hline & $(\mathrm{N}=$ & & $(\mathrm{N}=1$ & & $(\mathrm{N}=$ & \\
\hline Layperson CPR, n (\%) & 98 & (34.6) & $81^{*}$ & $(56.6)$ & 17 & (12.1) \\
\hline ROSC before hospital arrival, $\mathrm{n}(\%)$ & 31 & $(11.0)$ & 18 & (12.6) & 13 & (9.3) \\
\hline Hospital admission, n (\%) & 86 & (30.4) & 40 & $(28.0)$ & 46 & (32.9) \\
\hline Good neurological outcome, $n(\%)$ & 9 & (3.2) & 5 & (3.5) & 4 & (2.9) \\
\hline
\end{tabular}


Table 3 Variables associated with layperson CPR after EMS call

\begin{tabular}{|c|c|c|c|c|c|c|c|c|}
\hline \multirow[b]{2}{*}{ Variables } & \multirow{2}{*}{\multicolumn{2}{|c|}{$\begin{array}{l}\text { Layperson CPR } \\
\text { after EMS call } \\
(\mathrm{N}=98)\end{array}$}} & \multicolumn{3}{|c|}{ Univariate analysis } & \multicolumn{3}{|c|}{ Multivariate analysis } \\
\hline & & & OR & $95 \% \mathrm{Cl}$ & $\mathrm{p}$ Value & OR & $95 \% \mathrm{Cl}$ & p Value \\
\hline Men, n (\%) & 64 & $(64.3)$ & 1.20 & 0.73 to 1.99 & 0.48 & 1.24 & 0.67 to 2.28 & 0.51 \\
\hline Age $<75$ years old, $n(\%)$ & 46 & $(46.9)$ & 1.16 & 0.71 to 1.90 & 0.55 & 1.85 & 0.99 to 3.43 & 0.05 \\
\hline Victims with disabilities in daily living, $n(\%)$ & 18 & (18.4) & 2.10 & 1.04 to 4.26 & 0.04 & 2.57 & 1.10 to 5.99 & 0.03 \\
\hline Cardiac arrest at home, $\mathrm{n}(\%)$ & 87 & (88.8) & 0.85 & 0.39 to 1.89 & 0.69 & 0.50 & 0.19 to 1.31 & 0.16 \\
\hline EMS dispatcher-assisted CPR instruction, $n(\%)$ & 81 & (82.7) & 9.45 & 5.16 to 17.3 & $<0.001$ & 11.0 & 5.72 to 21.2 & $<0.001$ \\
\hline
\end{tabular}

or separated from the victims and indicates difficulties for rescuers and dispatchers that need to be addressed in order to develop dispatch-instructions.

Second, although we excluded the cases that a layperson witnessed CA after the call, due to the nature of prehospital CA study, we cannot deny the possibility to include victims not yet in CA before EMS arrival.

Third, since data of the abnormal breathing described by laypersons were derived from documents of CA written by each EMS dispatcher who actually took the emergency calls, some recall biases might exist.

Finally, since we investigated a small numbers of study subjects, the power to detect relationships of possible agonal respiration with clinical outcomes such as return of spontaneous circulation or hospital admission and survival is quite limited.

\section{CONCLUSION}

This population-based study revealed that $60 \%$ of CA victims had agonal respiration at the time of EMS call and lay rescuers' descriptions on breathing status were varied. Although EMS dispatch-instruction was associated significantly with an increase in layperson CPR, abnormal breathing was associated with a much lower rate of CPR instruction and, in turn, was related to a much lower rate of bystander CPR. These results indicate that EMS dispatchers should be more assertive for those CA victims with possible agonal respiration.

Acknowledgements We thank Fire Head Offices in Nara prefecture for provision of study information.

Contributors $\mathrm{HF}, \mathrm{MI}, \mathrm{TS}, \mathrm{YK}$ and KN performed most of the data collection. KS and NK performed statistical analysis of the collected data. HF, YU, MH, KN and TI performed interpretation of the data. $\mathrm{HF}$, TI and KO performed manuscript preparation. All authors read and approved this version of the manuscript to be submitted.

Funding This study was supported by the Foundation for Ambulance Service Development (Tokyo, Japan).

\section{Competing interests None.}

Ethics approval Ethical committee of Nara Medical University.

Provenance and peer review Not commissioned; externally peer reviewed.

Open Access This is an Open Access article distributed in accordance with the Creative Commons Attribution Non Commercial (CC BY-NC 3.0) license, which permits others to distribute, remix, adapt, build upon this work non-commercially, and license their derivative works on different terms, provided the original work is properly cited and the use is non-commercial. See: http://creativecommons.org/licenses/by-nc/3.0/

\section{REFERENCES}

1 Nichol G, Thomas E, Callaway CW, et al. Regional variation in out-of-hospital cardiac arrest incidence and outcome. JAMA 2008;300:1423-31.

2 Iwami T, Nichol G, Hiraide A, et al. Continuous improvements in "chain of survival" increased survival after out-of-hospital cardiac arrests: a large-scale population-based study. Circulation 2009;119:728-34.

3 Holmberg M, Holmberg S, Herlitz J, et al. Survival after cardiac arrest outside hospital in Sweden. Swedish Cardiac Arrest Registry. Resuscitation 1998;36:29-36.
4 Billittier AJt, Lerner EB, Tucker W, et al. The lay public's expectations of prearrival instructions when dialing 9-1-1. Prehosp Emerg Care 2000;4:234-7.

5 Rea TD, Eisenberg MS, Becker LJ, et al. Temporal trends in sudden cardiac arrest: a 25-year emergency medical services perspective. Circulation 2003;107:2780-5.

6 Bang A, Herlitz J, Martinell S. Interaction between emergency medical dispatcher and caller in suspected out-of-hospital cardiac arrest calls with focus on agonal breathing. A review of 100 tape recordings of true cardiac arrest cases. Resuscitation 2003;56:25-34.

7 Bohm K, Rosenqvist M, Hollenberg J, et al. Dispatcher-assisted telephone-guided cardiopulmonary resuscitation: an underused lifesaving system. Eur J Emerg Med 2007; 14:256-9.

8 Berdowski J, Beekhuis F, Zwinderman $\mathrm{AH}$, et al. Importance of the first link: description and recognition of an out-of-hospital cardiac arrest in an emergency call. Circulation 2009;119:2096-102.

9 Hauff SR, Rea TD, Culley LL, et al. Factors impeding dispatcher-assisted telephone cardiopulmonary resuscitation. Ann Emerg Med 2003;42:731-7.

10 Kuisma M, Hiltunen T, Maatta T, et al. Analysis of multiple casualty incidents-a prospective cohort study. Acta Anaesthesiol Scand 2005;49:1527-33.

11 Clark JJ, Larsen MP, Culley LL, et al. Incidence of agonal respirations in sudden cardiac arrest. Ann Emerg Med 1992;21:1464-7.

12 Cummins RO, Chamberlain DA, Abramson NS, et al. Recommended guidelines for uniform reporting of data from out-of-hospital cardiac arrest: the Utstein Style. Task Force of the American Heart Association, the European Resuscitation Council, the Heart and Stroke Foundation of Canada, and the Australian Resuscitation Council. Ann Emerg Med 1991;20:861-74.

13 Jacobs I, Nadkarni V, Bahr J, et al. Cardiac arrest and cardiopulmonary resuscitation outcome reports: update and simplification of the Utstein templates for resuscitation registries: a statement for healthcare professionals from a task force of the International Liaison Committee on Resuscitation (American Heart Association, European Resuscitation Council, Australian Resuscitation Council, New Zealand Resuscitation Council, Heart and Stroke Foundation of Canada, InterAmerican Heart Foundation, Resuscitation Councils of Southern Africa). Circulation 2004;110:3385-97.

14 Bobrow BJ, Zuercher M, Ewy GA, et al. Gasping during cardiac arrest in humans is frequent and associated with improved survival. Circulation 2008;118:2550-4.

15 Kuisma M, Boyd J, Vayrynen T, et al. Emergency call processing and survival from out-of-hospital ventricular fibrillation. Resuscitation 2005;67:89-93.

16 Flynn J, Archer F, Morgans A. Sensitivity and specificity of the medical priority dispatch system in detecting cardiac arrest emergency calls in Melbourne. Prehosp Disaster Med 2006;21:72-6.

$17 \mathrm{Ma} \mathrm{MH}$, Lu TC, Ng JC, et al. Evaluation of emergency medical dispatch in out-of-hospital cardiac arrest in Taipei. Resuscitation 2007;73:236-45.

18 Vaillancourt C, Charette ML, Bohm K, et al. In out-of-hospital cardiac arrest patients, does the description of any specific symptoms to the emergency medical dispatcher improve the accuracy of the diagnosis of cardiac arrest: a systematic review of the literature. Resuscitation 2011;82:1483-9.

19 Hallstrom AP, Cobb LA, Johnson E, et al. Dispatcher assisted CPR: implementation and potential benefit. A 12-year study. Resuscitation 2003;57:123-9.

20 Roppolo LP, Westfall A, Pepe PE, et al. Dispatcher assessments for agonal breathing improve detection of cardiac arrest. Resuscitation 2009;80:769-72.

21 Rea TD, Eisenberg MS, Culley LL, et al. Dispatcher-assisted cardiopulmonary resuscitation and survival in cardiac arrest. Circulation 2001;104:2513-16.

22 Bang A, Gustavsson M, Larsson C, et al. Are patients who are found deeply unconscious, without having suffered a cardiac arrest, always breathing normally? Resuscitation 2008;78:116-18.

23 Coons SJ, Guy MC. Performing bystander CPR for sudden cardiac arrest: behavioral intentions among the general adult population in Arizona. Resuscitation 2009;80:334-40.

24 White L, Rogers J, Bloomingdale $\mathrm{M}$, et al. Dispatcher-assisted cardiopulmonary resuscitation: risks for patients not in cardiac arrest. Circulation 2010;121:91-7.

25 Haley KB, Lerner EB, Pirrallo RG, et al. The frequency and consequences of cardiopulmonary resuscitation performed by bystanders on patients who are not in cardiac arrest. Prehosp Emerg Care 2011;15:282-7. 